\title{
La signification du neuroenhancement dans la pratique médicale
}

Regula Ott ${ }^{a}$,

Nikola Biller-Andorno ${ }^{b}$

a M. Sc., Ph. D. Doctorante à l'Institut d'Ethique Biomédicale, Université de Zurich

b Prof. Dr med. Dr phil., Directrice de l'Institut d'éthique biomédicale, Université de Zurich

* Les références st trouveront sous www.bullmed.ch

$\rightarrow$ Numéro actuel ou $\rightarrow$ Archives $\rightarrow 2013 \rightarrow 13 / 14$
Correspondance:

Académie Suisse des Sciences

Médicales ASSM

Petersplatz 13

CH-4051 Bâle

mail[at]samw.ch
Le neuroenhancement (NE) - dans le sens d'une amélioration des capacités cognitives et émotionnelles chez des personnes en bonne santé au moyen de substances médicamenteuses - est un thème largement débattu en éthique médicale [2-6]*. Ce faisant, le respect de l'autonomie des demandeurs de produits de NE est en opposition avec les questions liées aux risques encourus et aux évolutions sociales problématiques (pouvant revêtir la forme d'une pression de rendement exagérée avec une désolidarisation croissante).

En même temps, il n'existe que peu de données empiriques sur les utilisateurs (potentiels) [7-11] et encore moins sur les fournisseurs (potentiels) [5, $12,13]$. Les médecins jouent un rôle clé, du moins lorsqu'il s'agit de préparations délivrées sur ordonnance. Il est donc primordial que leurs perceptions et perspectives soient intégrées dans le débat de société.

\section{L'étude NEpA}

Le but de l'étude «La signification du neuroenhancement pour les praticiennes et praticiens des domaines de la psychiatrie et de la médecine de premier recours» (NEpA) est de mieux comprendre les expériences et les positions des médecins confrontés - de facto ou au moins potentiellement - à des demandes de produits de neuroenhancement dans leur pratique [1]. Pour ce faire, 800 questionnaires ont été envoyés en Suisse alémanique et en Suisse romande, dont 393 nous ont été retournés (taux de réponses 24,7\%). 379 d'entre eux ont pu être utilisés pour l'évaluation (23,0\%).

L'échantillon comprenait 180 femmes (47,9\%) et 196 hommes $\left(52,1 \% ; \mathrm{n}_{\text {Total }}=376\right.$ en raison de valeurs manquantes). L'âge moyen s'élevait à 48 ans avec un écart de 32 à 69 ans. 208 médecins avaient un titre de spécialiste en "psychiatrie et psychothérapie», 116 d'entre eux en «médecine générale», 65 en «médecine interne» et deux personnes avaient un autre titre de spécialiste respectivement aucun titre. 19 personnes avaient deux titres $\left(\mathrm{n}_{\text {Total }}=374\right)$.

\section{Degré de connaissance des notions de «neuroenhancement» et de «doping cérébral»}

A la question «Connaissez-vous la notion de neuroenhancement?», la majorité des participants $(68,9 \%$, $\left.\mathrm{n}_{\text {Total }}=376\right)$ ont répondu par la négative. Par contre, $53,6 \%$ d'entre eux $\left(\mathrm{n}_{\text {Total }}=371\right)$ connaissaient la notion de «doping cérébral».
On entend par «Human Enhancement» les interventions médicales ou biotechnologiques dont l'objectif primaire n'est pas le traitement ou la prévention de maladies, mais I' «amélioration» de caractéristiques non pathologiques. Un groupe de travail des Académies Suisses des Sciences Médicales (ASSM) et des Sciences Humaines et Sociales (ASSH) a examiné minutieusement les questions éthiques soulevées par l'enhancement et publié ses analyses et recommandations dans une brochure intitulée «La médecine pour les personnes en bonne santé?» (www.academiessuisses.ch $\rightarrow$ Projets et thèmes). Des extraits de ce rapport paraissent à intervalles irréguliers dans le BMS. L'étude NEpA présentée dans ce document a été réalisée à la demande du groupe de travail et avec son soutien. Le texte intégral de l'étude a été publié dans Swiss Medical Weekly [1]*.

\section{Concept de maladie et de thérapie}

Dans la question concernant les critères retenus par les médecins pour qu'un trouble soit qualifié de pathologique, le premier critère retenu est celui du «poids subjectif de la souffrance» $(83,6 \%)$, suivi des «conséquences négatives sur la capacité de travail au quotidien» (76,5\%) (tabl. 1).

Face à un patient qui demande des médicaments non indiqués, la majorité des participants ont choisi, parmi les différentes affirmations proposées: «En principe, je ne fais pas de prescription sans indication. Mais si la souffrance est trop éprouvante et si le patient souhaite tenter tout ce qui est possible, il est possible que je fasse une prescription sans indication claire. Cela dépend toutefois de la substance, des contre-indications et des effets indésirables» $(67,0 \%$, $\left.\mathrm{n}=250 ; \mathrm{n}_{\text {Total }}=373\right) .28,4 \%$ des médecins $(\mathrm{n}=106)$

Membres du groupe de travail

Prof. Dr méd. Dr phil. Nikola Biller-Andorno (présidente) Prof. Dr méd. Anne-Françoise Allaz

Dr phil. Gaia Barazzetti (jusqu'en 2010)

lic. ès lettres Nadja Birbaumer, ASSH

PhD Susanne Brauer, NEK-CNE

Prof. Dr méd. Jürg Kesselring

Prof. Dr méd. lic. phil. Iris Ritzmann

Dr phil. Simone Romagnoli

Dr biol. Adrian Rüegsegger, TA-SWISS

lic. iur. Michelle Salathé, MAE, ASSM

PD Dr théol. Markus Zimmermann-Acklin 


\section{Tableau 1}

Lorsque vous devez décider si vous êtes en présence d'un trouble pouvant ou non être qualifié de pathologique, quels sont selon vous les critères décisifs (réponses multiples possibles):

\begin{tabular}{|c|c|c|c|c|}
\hline & Oui, $n(\%)$ & Non $n(\%)$, & Parfois, $n(\%)$ & Total (n) \\
\hline Le poids subjectif des souffrances & $317(83,6)$ & $8(2,1)$ & $50(13,2)$ & 375 \\
\hline $\begin{array}{l}\text { La classification selon un système } \\
\text { reconnu (par ex. ICD ou DSM) }\end{array}$ & $165(43,5)$ & $93(24,5)$ & $100(26,4)$ & 358 \\
\hline $\begin{array}{l}\text { L'objectivation au moyen de procédés } \\
\text { de laboratoire ou d'imagerie, en } \\
\text { particulier en médecine somatique }\end{array}$ & $165(43,5)$ & $70(18,5)$ & $117(30,9)$ & 352 \\
\hline $\begin{array}{l}\text { Les conséquences négatives sur la } \\
\text { capacité de travail au quotidien }\end{array}$ & $290(76,5)$ & $11(2,9)$ & $65(17,2)$ & 366 \\
\hline
\end{tabular}

\section{Tableau 2}

Que feriez-vous dans la situation esquissée?

\begin{tabular}{lcccc} 
& $\begin{array}{l}\text { Prescription } \\
(\%)\end{array}$ & $\begin{array}{l}\text { Uniquement en l'absence } \\
\text { d'alternatives thérapeu- } \\
\text { tiques }(\%)\end{array}$ & $\begin{array}{l}\text { Pas de pre- } \\
\text { scription (\%) }\end{array}$ & $\begin{array}{l}\text { Transfer } \\
(\%)\end{array}$ \\
\hline Etudiant & $49(15,3)$ & $180(54,4)$ & $161(49,2)$ & $24(7,8)$ \\
\hline Mère isolée & $83(25,6)$ & $176(52,9)$ & $137(41,4)$ & $14(4,5)$ \\
\hline Femme timide & $107(32,7)$ & $244(70,1)$ & $88(26,8)$ & $19(6,1)$ \\
\hline Scientifique & $54(16,5)$ & $52(16,1)$ & $193(54,1)$ & $35(10,7)$
\end{tabular}

\section{Tableau 3}

Avez-vous déjà été confronté à de telles demandes dans votre pratique?

\begin{tabular}{lllll} 
& Oui, $\mathbf{n}(\%)$ & Non, $\mathbf{n}(\%)$ & nTotal & Valeur $\mathbf{p}$ \\
\hline $\begin{array}{l}\text { Exemple 1: } \\
\text { Etudiant }\end{array}$ & $200(53,5)$ & $174(46,5)$ & 374 & .179 \\
$\begin{array}{l}\text { Exemple 2: } \\
\text { Mère isolée }\end{array}$ & $156(42,0)$ & $215(58,0)$ & 371 & $.002^{* *}$ \\
$\begin{array}{l}\text { Exemple 3: } \\
\text { Femme timide }\end{array}$ & $180(52,9)$ & $160(47,1)$ & 340 & .278 \\
$\begin{array}{l}\text { Exemple 4: } \\
\text { Scientifique }\end{array}$ & $75(21,7)$ & $270(78,3)$ & 345 & $.000^{* * *}$ \\
\hline
\end{tabular}

La valeur $p$ du test $X^{2}$ est indiquée dans la dernière colonne.

* $(p<.05) ;{ }^{* *}(p<.01) ; * * *(p<.001)$. Si les valeurs divergent de façon significative $(p<05)$, la valeur supérieure est indiquée en caractères gras. qui réduise un peu son besoin de sommeil pour profiter au mieux du temps qu'il lui reste.

Le deuxième exemple concerne une mère isolée de deux enfants en âge scolaire, qui depuis quelques semaines soigne à son domicile sa mère malade en phase terminale et qui a accumulé beaucoup de fatigue. Pour surmonter cette situation, elle demande un «stimulant» à son médecin.

Le troisième cas est celui d'une femme de 24 ans, très timide, qui manque de confiance en elle dans ses relations avec les hommes et aimerait vaincre sa timidité. Elle attend de son médecin un remède qui influence positivement son attitude crispée et craintive.

Le quatrième et dernier cas est celui d'un scientifique renommé, qui revient d'un congrès à l'étranger. Il souffre du décalage horaire et demande une prescription de Modafinil pour être à nouveau performant dans les plus brefs délais.

Après la description des différents cas, les médecins ont été interrogés sur leurs réactions dans les différentes situations présentées et les prescriptions envisageables - si prescription il y a (voir tabl. 2).

Les résultats montrent que la majorité des participants refuseraient de prescrire un médicament dans les quatre exemples de cas. Malgré cela, environ un tiers d'entre eux $(32,7 \%)$ auraient délivré une ordonnance à la femme timide et environ un quart $(25,6 \%)$ à la mère isolée.

Les réponses à la question suivante: «Avez-vous déjà été confronté à de telles demandes dans votre pratique?» sont récapitulées dans le tableau 3. Environ la moitié des participants ont connu des situations telles que dans les exemples 1 et 3 . Un peu moins nombreux étaient ceux qui ont fait l'expérience du deuxième cas (42\%) et seulement $20 \%$ de celui du scientifique renommé. Néanmoins, les demandes restent peu fréquentes, dans la majorité des cas, 1 à 2 fois par an.

\section{Attitude personnelle}

De nombreux médecins participant à l'étude n'ont pas pu se décider pour ou contre le rejet fondamental du NE (41,1\%, nTotal=353). En revanche, la majorité d'entre eux a adhéré à l'idée que l'utilisation du NE dépendait de la situation et qu'ils ne le rejetteraient pas systématiquement $\left(49,0 \%, \mathrm{n}_{\text {Total }}=349\right)$. Seulement une minorité approuverait un modèle dans lequel la décision médicale pour la prescription d'une préparation serait basée sur les souhaits du patient $(9,6 \%$, $\mathrm{n}_{\text {Total }}=344$ ).

Pour déterminer les facteurs ayant influencé une éventuelle prescription dans les exemples cités, les six critères suivants, élaborés sur la base d'interviews avec des médecins du domaine clinique, ont été proposés aux médecins: 1) le poids subjectif de la souffrance, 2) la limitation temporelle de la prise, 3) la prévention d'une aggravation, 4) la responsabilité du patient à sa propre situation, 5) l'importance et la valeur accordées au désir de rétablissement et 6 ) les efforts visibles

\section{Les exemples de cas}

Quatre exemples de cas ont été présentés aux participants. Le premier exemple concerne un étudiant avant ses derniers examens décisifs; pour la «dernière ligne droite», il demande au médecin un médicament

ont répondu: «Si je ne peux pas poser d'indication haitée et s'il persiste dans sa demande, qu'il l'essaie. $71,6 \%$ des participants sont en principe ouverts à la prescription de neuroenhancer. 
d'avoir essayé par ses propres moyens. La majorité des médecins questionnés considéraient les trois premiers critères - le poids subjectif de la souffrance, la limitation temporelle de la prise et la prévention d'une aggravation - comme pertinentes (valeurs entre 75\% et $88 \%$ et entre $26 \%$ et $47 \%$ pour les trois autres affirmations).

A la fin du questionnaire, les médecins étaient priés d'évaluer trois affirmations. La première, «Chaque médecin doit décider lui-même s'il veut pratiquer le NE ou non» a été approuvée par la majorité d'entre eux (30\% ont qualifié l'affirmation d'assez juste, $24,5 \%$ de tout à fait juste, $\mathrm{n}_{\text {Total }}=367$ ). La deuxième proposition, selon laquelle «les pratiques de neuroenhancement sont une réalité. Puisqu'il en est ainsi, elles devraient faire partie de l'activité médicale pour le bien-être du demandeur. De cette façon, l'évaluation et la supervision des risques et des effets secondaires peuvent être garanties», a laissé environ un quart d'entre eux $(23,4 \%)$ indécis; le reste des réponses était réparti presque à parts égales entre l'adhésion $(39,4 \%)$ et le rejet $(37,2 \%)$. La troisième et dernière affirmation stipule que, si les médecins s'éloignent de plus en plus de leur mission originelle qui consiste à soigner les maladies, leur image serait ternie et la confiance de leurs patients altérée; celle a recueilli l'approbation de la majorité des participants $(64,6 \%)$.

Des analyses de groupes répartis selon la langue (français/allemand) et le sexe ont révélé que les participants francophones ont choisi plus souvent le poids subjectif des souffrances comme critère pour déterminer si un trouble est pathologique $(\mathrm{p}=0,012)$; elles ont également révélé que les femmes ont adhéré plus souvent que les homme à l'affirmation concernant l'érosion de la confiance du patient en son médecin $(\mathrm{p}=0,011)$.

\section{Limitations de l'étude}

Compte tenu du taux de réponses d'environ $25 \%$, d'éventuelles distorsions sont possibles. Ainsi, on pourrait penser que les médecins qui ont rempli le questionnaire sont surtout ceux qui affichent une position claire - qu'elle soit critique ou libérale - face au NE. A ceci s'oppose toutefois le fait que les résultats de cette étude coïncident avec ceux d'autres études déjà réalisées dans d'autres pays [par ex. 5]. Egalement des distorsions concernant des réponses socialement souhaitées sont plutôt improbables; non seulement en raison de l'anonymité soulignée à plusieurs reprises, mais également en raison de réponses douteuses d'un point de vue éthique et juridique (par ex. une prescription sans indication).

\section{Conclusions}

L'étude NEpA révèle que la plupart des médecins, qui ont pris part à l'étude, font preuve de retenue envers le NE, sans toutefois le rejeter fondamentalement. Leur décision dépend des alternatives, des conséquences attendues de la souffrance, de la sécurité du médicament et des préférences des demandeurs. Les critères objectifs concernant la maladie sont plutôt secondaires. Plus un état est pathologique (comme dans l'exemple de la jeune femme timide), plus le médecin est enclin à prescrire un médicament.

Il était surprenant de constater que, souvent, les notions de «neuroenhancement» et de «doping cérébral» étaient ignorées. Pourtant, comme le révèle l'étude, les médecins questionnés sont confrontés à de telles demandes dans leur pratique. Ce constat de même que l'hétérogénéité des réponses aux différentes affirmations morales dénoncent la nécessité d'un débat éthique approfondi. Un tel débat permettrait de refléter les incertitudes et ambivalences des questions éthiques liées au NE et de clarifier les positions personnelles et déontologiques. 


\section{Références}

1 Ott R, Lenk C, Miller N, Neuhaus Bühler R, BillerAndorno N. Neuroenhancement - perspectives of Swiss psychiatrists and general practitioners. Swiss Med Wkly. 2012;142:w13707.

2 Greely H, Sahakian B, Harris J, Kessler RC, Gazzaniga M, Campbell $\mathrm{P}$, et al. Towards responsible use of cognitive-enhancing drugs by the healthy. Nature. 2008;456: 702-5.

3 Lenk C. Kognitives Enhancement und das «Argument des offenen Lebensweges».

In: Schöne-Seifert B, Talbot D, Opolka U, Ach JS. Neuro-Enhancement. Ethik vor neuen Herausforderungen. Münster: Mentis-Verlag; 2008:93-106.

4 Outram SM. The use of methylphenidate among students: the future of enhancement? J Med Ethics. 2010;36:198-202.

5 Hotze TD, Shah K, Anderson EE, Wynia MK. «Doctor, Would You Prescribe a Pill to Help Me ...?» a national survey of physicians on using medicine for human enhancement. Am J Bioeth. 2011;11:3-13.

6 Savulescu J, ter Meulen R, Kahane G. Enhancing Human Capacities. Oxford: Wiley-Blackwell; 2011.

7 McCabe SE, Knight JR, Teter CJ, Wechsler H. Non-medical use of prescription stimulants among US college students: prevalence and correlates from a national survey. Addiction. 2005;100:96-106.
8Sahakian B, Morein-Zamir S. Professor's little helper. Nature. 2007;450:1157-9.

9Deutsche Angestellten-Krankenkasse (DAK). Gesundheitsreport 2009: Analyse der Arbeitsunfähigkeitsdaten. Schwerpunktthema Doping am Arbeitsplatz [Internet]. 2009 (cited 2012 May 31). www.dak.de/content/filesopen/ Gesundheitsreport_2009.pdf

10 Biedermann, F. Die Haltung der Deutschschweizer Bevölkerung zum pharmazeutischen cognitive enhancement. Hypothesen basierend auf einer postalischen Befragung in einer Beispielgemeinde. Dissertation. Universität Basel; 2011.

11 Franke AG, Bonertz C, Christ-

mann M, Huss M, Fellgiebel A, Hildt E, et al. Non-Medical Use of Prescription Stimulants and Illicit Use of stimulants for Cognitive Enhancement in Pupils and Students in Germany. Pharmacopsychiatry. 2011;44:60-6.

12 Bergström LS, Lynöe N. Enhancing concentration, mood and memory in healthy individuals: an empirical study of attitudes among general practitioners and the general population. Scand J Public Health. 2008;36:532-7.

13 Banjo OC, Nadler R, Reiner PB. Physician attitudes towards pharmacological cognitive enhancement: safety concerns are paramount. PLoS One. 2010;5:e14322. 